\title{
Sacubitril/valsartan: from the PARADIGM-HF trial results to heart failure patients in internal medicine. A narrative review
}

\author{
Valerio Verdiani \\ Internal Medicine Unit, Valdelsa Hospital, Siena, Italy
}

\begin{abstract}
Data regarding the treatment of heart failure (HF) patients derived from randomized, controlled clinical trials, which, with rare exceptions, appear to be distant from the real world of internal medicine. Many trials have been conducted in cardiology departments: however, the characteristics of patients admitted to cardiology wards are largely different from those of patients hospitalized in internal medicine wards. Recently, the PARADIGM-HF study established the efficacy of sacubitril-valsartan the first drug of the angiotensin II receptor neprilysin inhibitor (ARNI) class - versus enalapril in increasing survival and reducing hospitalizations in a selected population of HF patients with reduced ventricular function. Although practical guidance on the use of ARNI has been published, it is not specific to HF patients admitted to internal medicine wards. In this review, we examine all available data in order to understand if the characteristics of HF patients followed in internal medicine departments hinder or contraindicate the use of sacubitril-valsartan and what indications appear more appropriate in this setting.
\end{abstract}

\section{Introduction}

Heart failure (HF) is one of the major causes of morbidity and mortality in industrialized countries ${ }^{1}$ and - after vaginal delivery - represents the leading cause of hospitalization in Italy. ${ }^{2}$ Despite an improvement in treatments, about $50 \%$ of patients diagnosed with HF die within 5 years, ${ }^{3}$ a worse statistic than those for prostate, breast, and bowel cancer. ${ }^{4-6}$ Its prevalence - which in the general population is between $1 \%$ and $2 \%$, without any significant gender difference - increases with age, reaching 6.4\% over the age of 65 . Depending on the studies, the av-

Correspondence: Valerio Verdiani, Internal Medicine Unit, Valdelsa Hospital, Loc. Campostaggia, Poggibonsi, Siena, Italy.

E-mail: verdiani@fastwebnet.it

Key words: Heart failure; angiotensin receptor neprilysin inhibitor; therapy; elderly; hospitalized patients.

Conflict of interests: the author declares no potential conflict of interests.

Received for publication: 20 July 2020.

Revision received: 13 December 2020.

Accepted for publication: 13 December 2020.

This work is licensed under a Creative Commons Attribution NonCommercial 4.0 License (CC BY-NC 4.0).

${ }^{\circ}$ Copyright: the Author(s), 2021

Licensee PAGEPress, Italy

Italian Journal of Medicine 2021; 15:17-26

doi:10.4081/itjm.2021.1347 erage age of HF patients varies between 74 and 78 years. $^{7-10}$

Data regarding the treatment of HF patients derived from randomized, controlled clinical trials, which, with rare exceptions, appear to be distant from the real world of internal medicine. Patients enrolled are $70 \%$ males, around 62 years of age on average, and generally without comorbidities.

Recently, the PARADIGM-HF trial ${ }^{11}$ showed that treatment with sacubitril-valsartan - the first drug of the angiotensin II receptor neprilysin inhibitor (ARNI) class - in selected HF patients with reduced ventricular function reduced mortality by $20 \%$ and hospitalization for HF by $21 \%$ versus enalapril (Figure 1).

Although practical guidance on the use of ARNI has been published, it is not specific to HF patients admitted to internal medicine wards. ${ }^{12,13}$ Indeed, HF patients' hospitalization occurs for the majority of cases in internal medicine wards, while only $25 \%$ of them are treated in cardiology wards. ${ }^{14,15}$

The matter is not only quantitative but also qualitative because the characteristics of patients admitted to cardiology wards are substantially different from those of patients hospitalized in internal medicine wards.

In order to understand whether the characteristics of HF patients treated in internal medicine wards hinder or contraindicate the use of sacubitril-valsartan and to determine whether the indications appear more appropriate in this setting, we performed a Medline search. The search was limited to articles in English in the last 17 years and adult populations. We used the Title/Abstracts with the term 'Sacubitril Valsartan' and the following search strings: ('Sacubitril Valsartan') AND ('Internal Medicine OR 'Geriatric'); ('Heart Failure') AND ('Internal Medicine') AND ('Cardiol- 
ogy'). The literature search was completed by the analysis of related articles and a manual search.

\section{Heart failure in internal medicine: patients' characteristics}

The TEMISTOCLE study, conducted in Italy in 2000 , showed that $76.2 \%$ of HF patients in internal medicine departments were $>70$ years of age, $v s 56.3 \%$ in cardiology departments $(\mathrm{P}<0.0001)$ and that they presented a significantly greater incidence of comorbidities (73.6\% vs 64.5\%; $\mathrm{P}<0.0001) .{ }^{16}$ In a more recent Spanish study, HF patients admitted to internal medicine wards were remarkably older than those in cardiology wards ( 74 years $v s 68.5$ years; $\mathrm{P}=0.001$ ), had longer hospital stays ( 10.8 days $v s 8.7$ days; $\mathrm{P}=0.001$ ), and had more comorbidities, in particular atrial fibrillation (43.6\% vs 30.7\%; $\mathrm{P}=0.002$ ), chronic obstructive pulmonary disease (COPD) $(26.6 \%$ vs $10.4 \%$; $\mathrm{P}<0.001)$, renal failure $(30.3 \%$ vs $19.3 \% ; \mathrm{P}<0.01)$, and anemia $(41.9 \%$ vs $33.4 \% ; \mathrm{P}<0.05) .{ }^{17}$ In a Canadian study, HF patients admitted to internal medicine wards were older and had a greater number of comorbidities than those hospitalized in cardiology wards. ${ }^{18}$ In the SMIT study (an observational study performed on 770 patients admitted for HF to 32 departments of internal medicine in Tuscany, a region of central Italy), there was a female prevalence (F 55\%, M 45\%) and an average age of 82.5 years; $71.5 \%$ had at least three comorbidities, and $40.2 \%$ at least four comorbidities, in particular arterial hypertension, renal impairment, atrial fibrillation, COPD, and diabetes mellitus. ${ }^{19}$

Preserved left systolic ventricular function is more frequent in internal medicine wards than in cardiology wards. In the TEMISTOCLE study, $40.8 \%$ of patients admitted to internal medicine had ejection fraction (EF) $>40 \%$, vs $28.4 \%$ of those admitted to cardiology. In the Canadian study, EF $>55 \%$ was found in $51 \%$ of the patients in internal medicine and $34.8 \%$ of those in cardiology. However, severe impairment of the left ventricular function $(\mathrm{EF}<35 \%)$ was reported in approximately $20 \%$ of patients admitted to internal medicine wards.

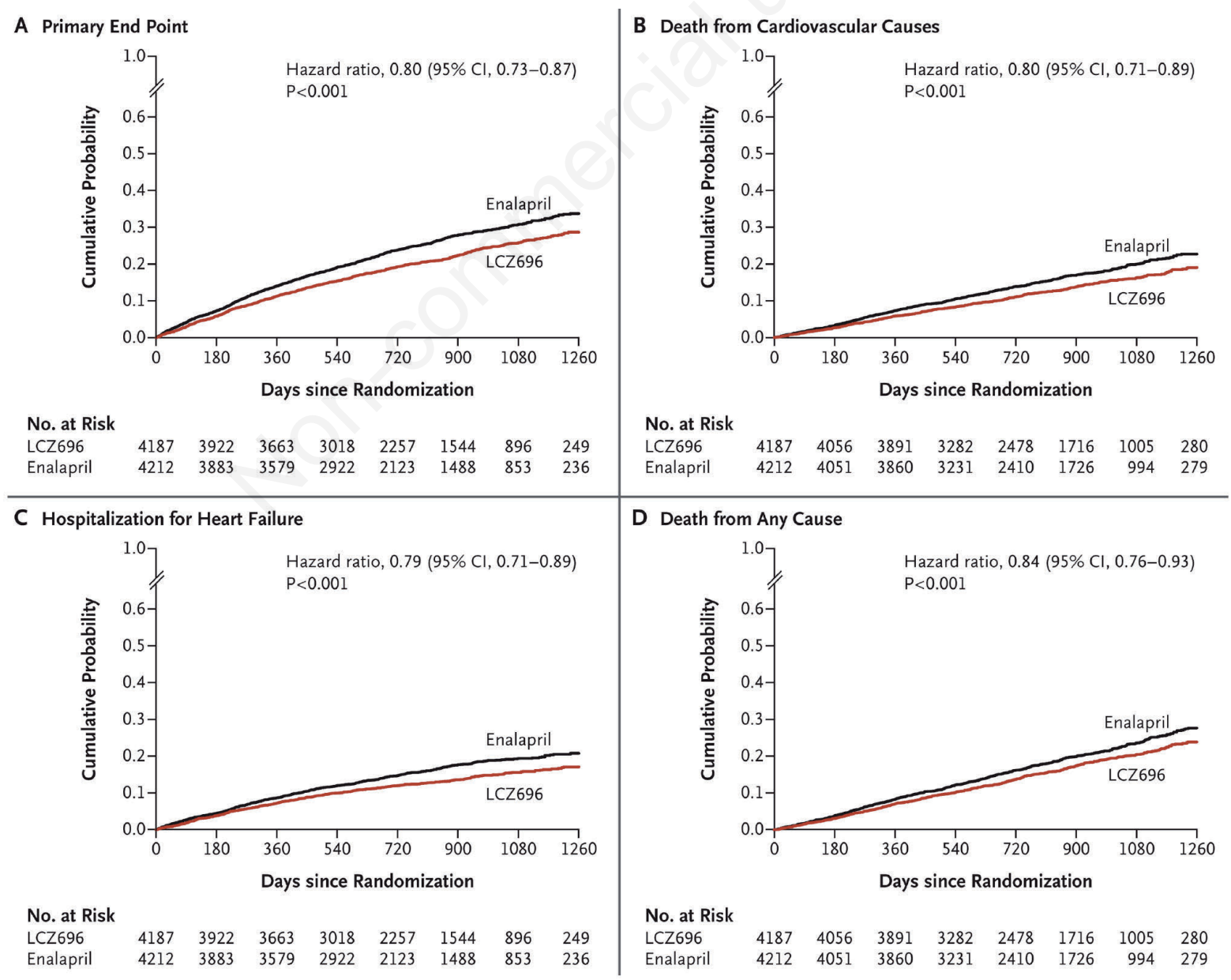

Figure 1. Kaplan-Meier curves for key study outcomes: PARADIGM-HF results. From: McMurray et al., $2014^{11}$ with permission. 


\section{Angiotensin II receptor neprilysin inhibitor: mechanism of action and guidelines}

The mechanism of action of the ARNI is twofold. Valsartan blocks angiotensin AT1 receptors, thereby inhibiting the effects of the activation of the renin-angiotensin system (RAAS). Sacubitril, which is rapidly converted to its active form, inhibits neprilysin, an enzyme that fragments natriuretic peptides (ANP, BNP, and $\mathrm{CNP}$ ), that increases their circulating concentrations. The block of the negative actions of angiotensin (e.g., vasoconstriction), together with the positive actions of the natriuretic peptides (e.g., vasodilation, natriuresis, RAAS modulation), are the basis of the efficacy of the drug (Figure 2).

Based on the results of the PARADIGM-HF study, the guidelines of the European Society of Cardiology (ESC) recommended the substitution of angiotensinconverting enzyme inhibitors (ACEI) or angiotensin receptor blockers (ARB) with sacubitril-valsartan in all patients with symptomatic heart failure (New York Heart Association [NYHA] classes II-IV), despite a therapy with ACEI or ARB, beta-blockers and miner- alocorticoid receptor antagonist (MRA) and $\mathrm{EF} \leq 35 \%$ (recommendation class I level A).$^{20}$ The guidelines of the American College of Cardiology (ACC), the American Heart Association (AHA), and the Heart Failure Society of America recommend the substitution of the ACEI or ARB with sacubitril-valsartan in symptomatic HF patients with reduced ventricular function (recommendation class I level B). ${ }^{21}$ The level $\mathrm{B}$ of the recommendation depends on the fact that the results come from a single trial, although it has been reported that the statistical significance of the PARADIGM-HF study ( $\mathrm{P}=0.0000004)$ was equivalent to 45 trials with $\mathrm{P}<0.05 .{ }^{22}$

\section{Angiotensin II receptor neprilysin inhibitor and elderly patients}

Patients with HF are increasingly older due to the progressive increase in average life expectancy and the efficacy of therapies.

In the PARADIGM-HF trial, despite the average age being 63.8 years, 1563 patients $(18.6 \%)$ were $\geq 75$ years old, and $587(7.0 \%)$ were $\geq 80$ years old. Com-

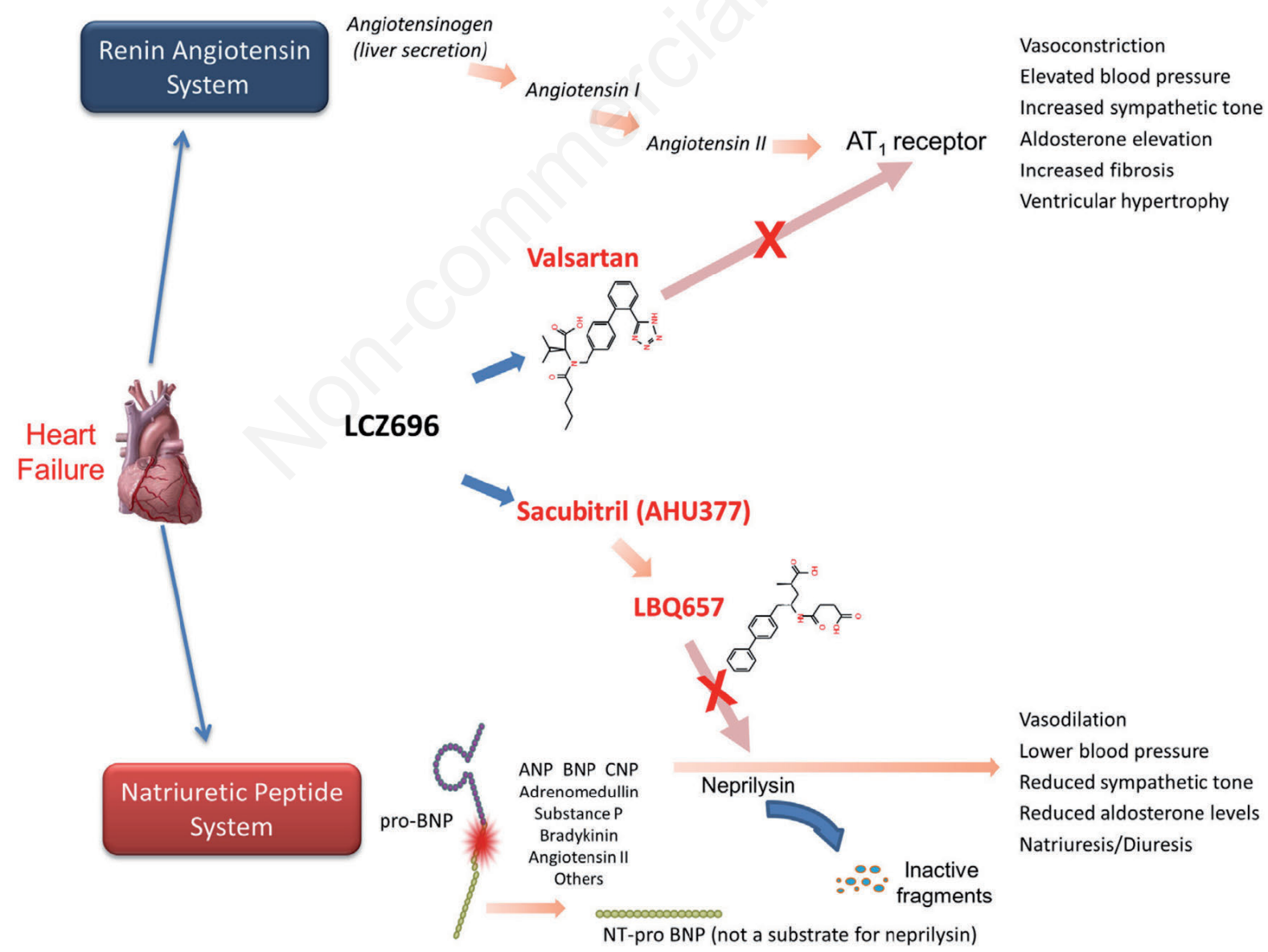

Figure 2. Mechanism of sacubitril-valsartan action. From: Vardeny et al. J Am Coll Cardiol HF 2014;2:663-70 with permission. 
pared with younger patients, the elderly were more frequently female, had more comorbidities, higher values of systolic blood pressure, creatinine, natriuretic peptide, and higher mean EF values; in other words, they more closely approximated the real-world setting of internal medicine. A retrospective analysis classified the entire cohort from the PARADIGM-HF trial based on age groups and found that also in the group of patients aged 65 to 74 years there was a significant risk reduction of $20 \%$ [hazard ratio (HR) $0.80 ; 95 \%$ confidence intereval (CI) $0.68-0.93$ ] for patients receiving sacubitril-valsartan versus enalapril in relation to the primary outcome (cardiovascular death or hospitalization for HF). ${ }^{23}$ Although it did not reach statistical significance, in the subgroup of patients $\geq 75$ years old, there was a trend towards risk reduction for the primary outcome (HR $0.86 ; 95 \%$ CI $0.72-1.04$ ) and the secondary outcomes (Figure 3).

Concerning safety, compared to younger patients, older patients showed a higher frequency of symptomatic hypotension both in the enalapril arm and in the sacubitril-valsartan arm. However, even in patients $\geq 75$ years of age, treatment discontinuation, for this reason, was minimal $(1.3 \%$ in the enalapril arm and
$1.8 \%$ in the sacubitril-valsartan arm). In patients $\geq 75$ years old, discontinuation of treatment due to worsening renal function was lower in the sacubitril-valsartan $\operatorname{arm}(0.6 \%)$ than in the enalapril arm $(2.1 \%)$.

These data are encouraging for the use of sacubitril-valsartan in elderly and very elderly patients. However, in the absence of large trials involving this type of population, we must use caution. Starting with small doses and ensuring close clinical checks should be considered.

Polypharmacy is another problem that internists must manage in elderly patients. Sacubitril-valsartan does not add to, but instead replaces ACEI or ARB, and this could have a favorable effect on compliance.

\section{Angiotensin II receptor neprilysin inhibitor and quality of life}

Improvement in quality of life and functional capacity, especially in elderly patients, is a reasonable outcome, even when it is impossible to expect a significant impact on mortality. This consideration is frequent in internal medicine wards. Furthermore,
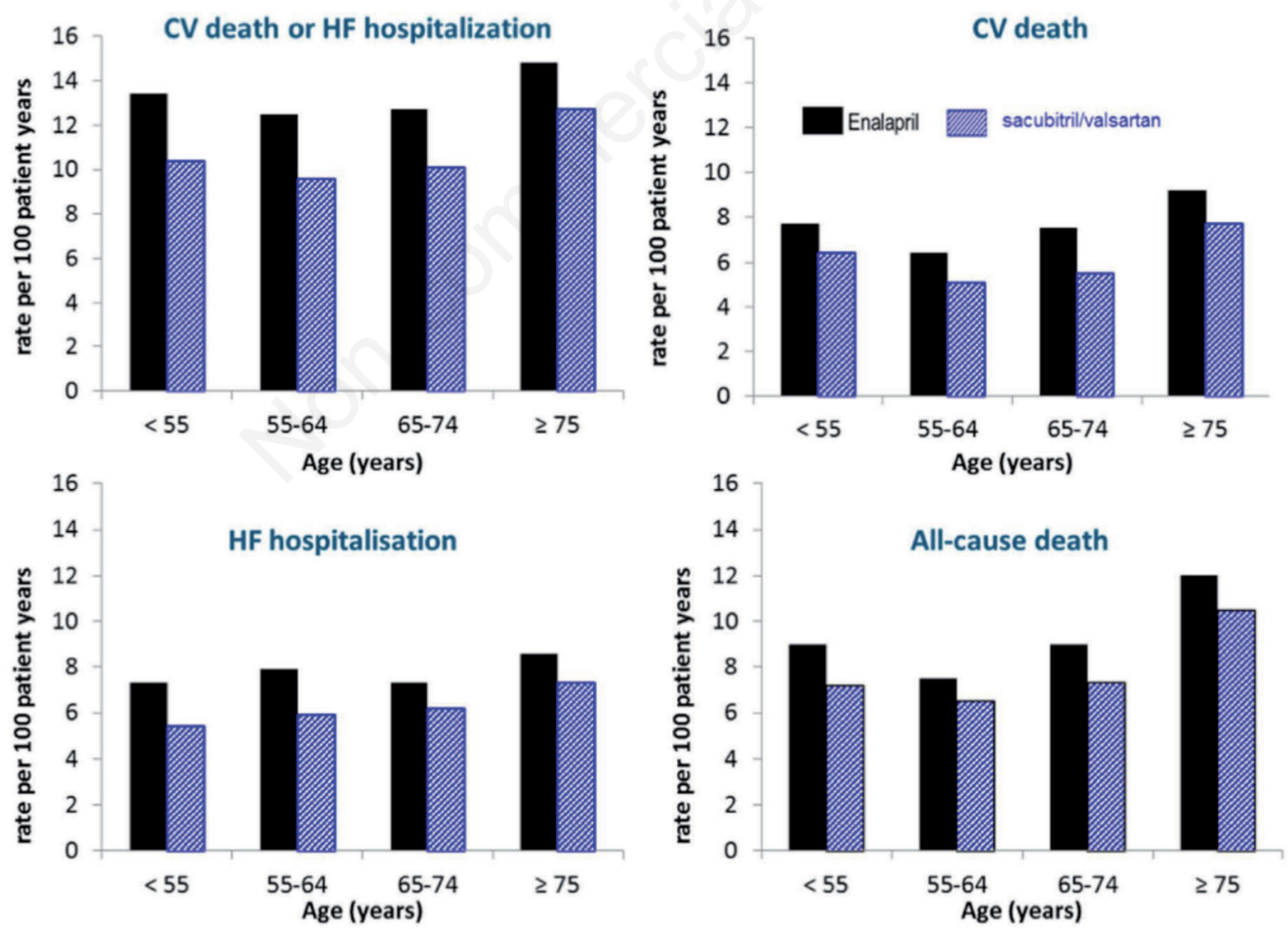

Figure 3. Effect of sacubitril-valsartan on the rate of primary endpoint and component and all-cause mortality in patients randomized in the PARADIGM-HF trial according to age. CV, cardiovascular; HF, heart failure. From Jhund and McMurray, $2016^{22}$ Freely available from Creative Commons (CC BY-NC 4.0). 
health-related quality of life in HF is worse than in several other chronic diseases.

Secondary analyses of the PARADIGM-HF studies have shown that the global scores of the international index, the Kansas City Cardiomyopathy Questionnaire (KCCQ), were superior in patients treated with sacubitril-valsartan compared to those treated with enalapril ${ }^{24}$ and that - in particular - a significant improvement in physical and social activity was obtained. ${ }^{25}$

\section{Angiotensin II receptor neprilysin inhibitor and renal impairment}

Renal failure is a major comorbidity in HF patients, particularly in those admitted to internal medicine wards. In a large cohort study of patients hospitalized for HF, $43.5 \%$ had stage III kidney failure (moderate renal dysfunction), and $13.1 \%$ had stage IV (severe renal dysfunction). ${ }^{26}$ In the aforementioned SMIT study, $71.9 \%$ of patients admitted to the internal medicine wards for HF had estimated glomerular filtration rate (eGFR) values $<60$ $\mathrm{mL} / \mathrm{min} / 1.73 \mathrm{~m}^{2}$ and $25.3 \%$ had eGFR values $<30$ $\mathrm{mL} / \mathrm{min} / 1.73 \mathrm{~m}^{2}$. We know that valsartan alone showed to have positive effects on renal function. Several studies have shown a reduction in albuminuria in patients with renal insufficiency treated with valsartan, and this effect was independent of the lowering of blood pressure values. ${ }^{27}$ Natriuretic peptides, in turn, determine an increase in eGFR values due to vasodilation of the afferent arteriole and concomitant vasoconstriction of the efferent arteriole. ${ }^{28}$ With ARNI it is, therefore, possible to potentially obtain a twofold favorable nephroprotection effect.

Indeed, in the PARAMOUNT study, sacubitrilvalsartan in HF patients with preserved EF resulted in a lower renal function reduction compared to that achieved by valsartan alone. ${ }^{29}$ In the TITRATION study, differences in creatinine level were negligible between more or less aggressive treatment with sacubitril-valsartan. ${ }^{30}$

In PARADIGM-HF, approximately $30 \%$ of enrolled patients had baseline eGFR values $<60$ $\mathrm{mL} / \mathrm{min} / 1.73 \mathrm{~m}^{2}$. The analysis of this subgroup of patients confirmed the greater efficacy of sacubitril-valsartan compared to enalapril to prevent clinical events, which was true for all endpoints. While not reaching statistical significance, there were fewer acute deterioration of renal function in the sacubitrilvalsartan arm, and fewer episodes of hyperkalemia. The values of eGFR were also higher than those in the enalapril arm. The increase in urinary albumin excretion detected in the sacubitril-valsartan arm tended to stabilize over time and did not influence the clinical outcomes. ${ }^{31}$
Although this analysis supports the prescription of sacubitril-valsartan even in patients with renal failure, careful monitoring should be arranged in patients with eGFR slightly above $30 \mathrm{~mL} / \mathrm{min} / 1.72 \mathrm{~m}^{2}$, bearing in mind that a temporary worsening of renal function could occur after the start of treatment. In such cases, the absence of nephrotoxic elements (e.g., nonsteroidal anti-inflammatory drugs) should be verified and act on possible dehydration (e.g., reduction of diuretic and/or fluid intake) before decreasing the dose of sacubitril-valsartan. Instead, drug discontinuation is appropriate when eGFR is $<15 \mathrm{~mL} / \mathrm{min} / \mathrm{m}^{2}$.

\section{Angiotensin II receptor neprilysin inhibitor and diabetes mellitus}

In internal medicine departments, diabetes mellitus is present in about one-third of patients hospitalized for HF. ${ }^{32}$ Also, in the PARADIGM-HF study, $34.7 \%$ of patients in the sacubitril-valsartan arm and $34.6 \%$ of those in the enalapril arm had diabetes. A post hoc analysis of these patients demonstrated significant advantages of the ARNI treatment. ${ }^{33}$ After 12 months of treatment, glycated hemoglobin was reduced by $0.26 \%$ in the sacubitril-valsartan arm, versus $0.16 \%$ in the enalapril arm $(\mathrm{P}=0.002)$, with similar results at Year 2 and 3. Moreover, the switch to - or the initiation of - insulin therapy was $29 \%$ lower in the sacubitril-valsartan arm, and the use of oral anti-diabetics was also lower. The reason for this beneficial effect on diabetes mellitus is not clear. Better cardiac performance may provide a beneficial global effect on the metabolism, and, more directly, the inhibition of neprilysin modulates its circulating substrates, including glucagon-like peptide 1 , increases insulin sensitivity. ${ }^{34-37}$

\section{Angiotensin II receptor neprilysin inhibitor and systolic blood pressure}

HF patients often have low values of systolic blood pressure (SBP). Hypotension is a negative prognostic factor, ${ }^{38}$ and internists are generally concerned about prescribing medications that tend to further lower SBP, particularly in elderly patients. Analyses of the PARADIGM-HF data concerning the efficacy and safety of sacubitril-valsartan versus enalapril, according to SBP baseline levels and SBP values after randomization, ${ }^{39}$ showed that sacubitril-valsartan improved the clinical outcomes for all SBP values. Even in patients with persistent low SBP values after treatment, sacubitril-valsartan was superior to enalapril in reducing mortality and morbidity. While it is true that patients with lower SBP values had a greater number of adverse events, this occurred regardless of the assigned treatment arm. Pa- 
tients who showed a higher frequency of hypotensive episodes in the PARADIGM-HF trial were older and had lower pressure values upon randomization; however, the benefits of sacubitril/valsartan were similar to those of patients who had not experienced symptomatic hypotension. ${ }^{40}$

Hypotension is one of the main side effects of sacubitril-valsartan, and treatment should not be initiated with SBP values $<100 \mathrm{mmHg}$ (criterion of exclusion from the PARADIGM-HF trial). Considering the beneficial effects of sacubitril-valsartan also in persistent low SBP value, if the SBP values reduce during treatment, the other possible determining conditions should be evaluated before stopping the medication. We should consider that treatment with sacubitril-valsartan frequently allows individualized dose reductions of loop diuretic doses. ${ }^{41}$ In case of hypotension risk, the use of bisoprolol as the beta-blocker of choice, the suspension of calcium-antagonists and nitrates, and tamsulosin substitution with finasteride (in case of treatment for prostatic hypertrophy) should be considered.

Finally, as expected, sacubitril/valsartan versus enalapril benefits were also confirmed for patients with $\mathrm{SBP} \geq 140 \mathrm{mmHg}$.

\section{Angiotensin II receptor neprilysin inhibitor and Alzheimer's disease}

There is considerable overlap between the populations suffering from HF and Alzheimer's disease (AD) ${ }^{42}$ Cognitive defects were found in $30 \%$ of HF patients admitted to internal medicine wards. ${ }^{19}$ Neprilysin is responsible for the breakdown of peptides, including amyloid-beta peptide. It has been hypothesized that inhibiting neprilysin may elevate levels of this peptide in the brain. ${ }^{43}$ However, when sacubitril-valsartan was administered to healthy human volunteers for two weeks, no changes were noted in the cerebrospinal fluid levels of amyloid-beta ${ }^{44}$ The PARADIGM-HF trial excluded patients with $\mathrm{AD}$ and did not evaluate cognitive function; however, the trial included reports of dementia-related adverse effects, and there were no differences reported between the treatment groups.

Nevertheless, it is difficult to say if the trial duration would have been sufficient to evaluate cognitive function. Currently, although some authors suggest that careful monitoring should occur in patients who may be affected by $\mathrm{AD},{ }^{45}$ there is no evidence as to the deleterious effects of sacubitril/valsartan on cognitive function or the progression of AD. Furthermore, we should consider that the cognitive decline in HF patients may not be the result of the only $\mathrm{AD}$, but could also be due to vascular abnormalities and decreased cardiac function ${ }^{46}$ and that another contributor to the cognitive impairment in HF patients is unplanned hospital admission due to decompensation. ${ }^{47}$

\section{Angiotensin II receptor neprilysin inhibitor and hyperkalemia}

The PARADIGM-HF trial showed no statistically significant difference in the frequency of hyperkalemia (serum potassium $>5.5 \mathrm{mmol} / \mathrm{L}$ ) in the sacubitril-valsartan arm versus the enalapril one. In both arms, hyperkalemia led to the discontinuation of treatment in rare cases $(<1 \%)$. Although the guidelines recommend using MRAs in symptomatic HF patients with EF $\leq 35 \%$, in clinical practice, this prescription occurs only in $18 \%-56 \%$ of eligible patients. ${ }^{48-52}$ In internal medicine departments, the prescription of MRAs upon discharge occurs in about $40 \%$ of the patients admitted for heart failure. ${ }^{53}$ The reason for this degree of under-treatment is not well known; however, the fear of hyperkalemia likely plays a predominant role, especially when renal insufficiency is present..$^{54,55}$ However, a subanalysis of the PARADIGM-HF trial found that severe hyperkalemia (serum potassium $>6 \mathrm{mmol} / \mathrm{L}$ ) was present more often in the enalapril arm than in the sacubitril-valsartan arm (3.1 vs 2.2 for 100 patient-years; HR 1.37 [95\% CI 1.06-1.76]; $\mathrm{P}=0.02$ ), suggesting that neprilysin would attenuate the risk of hyperkalemia when MRAs are combined with other inhibitors of the renin-angiotensin-aldosterone system. ${ }^{5}$

\section{Angiotensin II receptor neprilysin inhibitor and hyperuricemia}

Although the effect of hyperuricemia on cardiovascular events is not yet well understood, an association between hyperuricemia and a worse prognosis has been reported in patients with chronic ${ }^{57}$ and acute ${ }^{58}$ HF. An inverse relationship was also observed between serum uric acid levels and EF in elderly male patients with HF. ${ }^{59}$ In the PARADIGM-HF trial, patients with higher uric acid values had a worse prognosis than those with low values. Patients in the sacubitril-valsartan arm achieved a $24 \%$ reduction in serum uric acid compared to those in the enalapril arm, although the prognosis improvement was independent of such reduction. ${ }^{60}$

\section{Angiotensin II receptor neprilysin inhibitor and target dose}

In the PARADIGM-HF trial, the benefits of treatment with sacubitril-valsartan were significant in patients who already took ACEI/ARB and beta-blockers at target doses. However, in internal medicine's clinical practice, fewer patients are treated with ACEI or ARB than in cardiology practices. ${ }^{18}$ Furthermore, due to age and comorbidities, it is rare to reach the target dose. It is likely that some HF patients admitted to internal med- 
icine departments will not be able to achieve the target dose of sacubitril-valsartan (200 mg bid) as well.

Although found in a post hoc analysis ${ }^{61}$ the reduction of sacubitril-valsartan drug dosage continues to maintain a relative benefit versus enalapril. Another interesting aspect is that sacubitril-valsartan retains its advantages even in patients who do not receive the recommended concomitant treatments (e.g., betablockers, MRAs). ${ }^{62}$

In the TITRATION study, ${ }^{30}$ the initiation of sacubitril-valsartan at low doses (24/26 mg bid) with a slower up-titration $(24 / 26 \mathrm{mg}$ bid for 2 weeks, then $49 / 51 \mathrm{mg}$ bid for 3 weeks, then 97/103 mg bid) showed the same tolerability profile compared to the approach followed in PARADIGM-HF (initiation with $49 / 51$ bid for 2 weeks, then $97 / 103$ bid) and at the end of the study reached the same proportion of patients taking the maximum dose.

In patients naïve to ACEI or ARB, or in those who did not take them at the target dose, the more cautious approach (low initial dose and slower up-titration) made it easier to reach the target dose and to maintain it, mainly due to fewer episodes of hypotension, hyperkalemia, and renal dysfunction.

Therefore, this approach is indicated in the majority of HF patients hospitalized in internal medicine departments (Table 1).

\section{Angiotensin II receptor neprilysin inhibitor in hospitalized patients}

The subjects enrolled in the PARADIGM-HF trial were outpatients; therefore, it is unclear whether the study results could be generalized to patients hospitalized for acute HF. Recently, the results of the PIONEER-HF study have been published. ${ }^{63}$ It enrolled a population of 881 patients hospitalized for HF with reduced ventricular function. After hemodynamic stabilization (SBP of at least $100 \mathrm{mmHg}$ for the previous 6 hours, no dose increase of intravenous diuretics, no use of intravenous vasodilators in the previous 6 hours, and no intravenous use of inotropic agents during the previous 24 hours), patients were randomly assigned to receive sacubitril-valsartan or enalapril before hospital discharge. The results showed that the pre-discharge initiation of sacubitril-valsartan led to a more significant reduction in the concentration of $\mathrm{N}-$ terminal pro-B-type natriuretic peptide (NT-proBNP: a marker of both neuro-hormonal hyperactivity and congestion) compared to enalapril over an 8-week follow-up. The worsening renal function rate, hyperkalemia, symptomatic hypotension, and angioedema did not differ significantly between the two arms. A secondary combined clinical endpoint also demonstrated marked improvement in the sacubitril-valsartan arm compared to enalapril, mainly resulting from a robust reduction in the number of re-hospitalizations.

The TRANSITION study ${ }^{64}$ that enrolled about 1000 patients with a 10-week follow-up also demonstrated that the initiation of sacubitril-valsartan in patients hospitalized for HF after stabilization (SBP $\geq 110$ $\mathrm{mm} \mathrm{Hg}$ for at least 6 hours, not having received IV diuretics or vasodilators for at least 24 hours, except for nitrates or inotropic agents at the time of hospitalization), compared to a post-discharge initiation within two weeks, allowed the achievement of statistically equivalent percentages of the target dose (primary endpoint) or other dosages, with a limited drop-out rate that was equivalent in the two arms.

The results of the Pioneer-HF study and Transition-HF studies demonstrated safety in initiating sacubitril-valsartan during hospitalization in stable HF patients. In clinical practice, this approach is probably more appropriate when a post-discharge program is possible.

Table 1. Starting dose and dose titration for sacubitril-valsartan in a variety of patient populations with heart failure and reduced ejection fraction.

\begin{tabular}{|c|c|c|}
\hline Population with HFrEF & $\begin{array}{l}\text { Starting dose of sacubitril/ } \\
\text { valsartan }\end{array}$ & Uptitration and target dos \\
\hline No patient characteristics requiring caution or dose reduction & $49 \mathrm{mg} / 51 \mathrm{mg}$ twice daily & $\begin{array}{l}\text { Uptitration by doubling of dose every } 2-4 \text { weeks } \\
\text { until dose of } 97 \mathrm{mg} / 103 \mathrm{mg} \text { twice daily is reached }\end{array}$ \\
\hline $\begin{array}{l}\text { Currently only taking a low target dose of ACE inhibitor or } \\
\text { ARB* }\end{array}$ & $24 \mathrm{mg} / 26 \mathrm{mg}$ twice daily & \\
\hline No ACE inhibitor or ARB in the past & $24 \mathrm{mg} / 26 \mathrm{mg}$ twice daily & \\
\hline $\mathrm{eGFR}<30 \mathrm{~mL} / \mathrm{min} / \mathrm{m}^{2 \circ}$ & $24 \mathrm{mg} / 26 \mathrm{mg}$ twice daily & \\
\hline Moderate hepatic impairment (Child-Pugh class B) & $24 \mathrm{mg} / 26 \mathrm{mg}$ twice daily & \\
\hline Elderly & $24 \mathrm{mg} / 26 \mathrm{mg}$ twice daily & \\
\hline
\end{tabular}




\section{Conclusions}

Recently, based on PARADIGM-HF trial results, European and American guidelines recommended the substitution of ACEIs or ARBs with ARNIs in symptomatic HF patients with reduced ventricular function. In that study, the administration of sacubitril-valsartan - the first drug of the ARNI class - demonstrated increased survival and reduced hospitalizations for $\mathrm{HF}$ compared to patients receiving enalapril. The population enrolled in the study had an overall average age of around 64 years, with $80 \%$ male patients. It included only stable outpatients treated with beta-blockers and ACEIs or ARBs and with eGFR $>30 \mathrm{~mL} / \mathrm{min}$. Therefore, the transfer of the results to the real-world setting of HF patients, particularly those treated in internal medicine wards, has been the subject of discussion, and much research has been done to look for adequate answers.

The majority of HF patients are hospitalized in internal medicine departments. Observational studies show that they have different characteristics from those admitted to cardiology departments. In particular, they are older and have a more significant number of comorbidities.

All the post hoc analyses carried out have shown no contraindications to the use of sacubitril-valsartan in this type of patient. Indeed, beneficial aspects have been found in terms of both renal and metabolic function. In addition, even when long survival is not expected, sacubitril-valsartan versus enalapril showed significant improvements in quality of life.

The fact that results regarding HF patients mainly cared for by internists arise from sub-analysis of trials or studies with small populations should suggest caution. Pending broader and more specific trials in complex, frail, and elderly patients, it is prudent to start sacubitril-valsartan at low doses, with slower up-titration, modulation of concomitant treatment, and careful follow-up.

In conclusion, even in patients treated in internal medicine departments, the substitution of ACEIs or ARBs with ARNIs in symptomatic HF patients with reduced ventricular function appears to be safe and effective while offering benefits to the quality of life and some concomitant comorbidities. Special attention is required for frail and elderly patients.

\section{References}

1. Swedberg K, Cleland J, Dargle H, et al. Guidelines for the diagnosis and treatment of chronic heart failure: executive summary (update 2005). The task force for the diagnosis and treatment of chronic heart failure of the European Society of Cardiology. Eur Heart J 2005;26:1115-40.
2. Ministero della Salute. Rapporto annuale sull'attività di ricovero ospedaliero (Dati SDO 2016). Available from: http://www.salute.gov.it/portale/documentazione/p6

3. Lewis KS, Butler J, Bauersachs J, Sandner P. The threedecade long journey in heart failure drug development. Handb Exp Pharmacol 2017;243:1-14.

4. Brenner H, Bouvier AM, Foschi R, et al. Progress in colorectal cancer survival in Europe from the late 1980s to the early 21 st century: the EUROCARE study. Int J Cancer 2012;131:1649-58.

5. Coleman MP, Forman D, Bryant H, et al. Cancer survival in Australia, Canada, Denmark, Norway, Sweden, and the UK, 1995-2007 (the International Cancer Benchmarking Partnership): an analysis of populationbased cancer registry data. Lancet 2011;377:127-38.

6. Siegel R, DeSantis C, Virgo K, et al. Cancer treatment and survivorship statistics, 2012. CA Cancer J Clin 2012;62:220-41.

7. Murdoch DR, Love MP, Robb SD, et al. Importance of heart failure as a cause of death: changing contribution to overall mortality and coronary heart disease mortality in Scotland 1979-1992. Eur Heart J 1998;19:1829-35.

8. Cleland JG, Gemmell I, Khand A, Boddy A. Is the prognosis of heart failure improving? Eur J Heart Fail 1999;1:229-41.

9. Wilhelmsen L, Rosengren A, Eriksson H, Lappas G. Heart failure in the general population of men-morbidity, risk factors and prognosis. J Intern Med 2001;249:253-61.

10. Cowie MR, Wood DA, Coats AJ, et al. Incidence and aetiology of heart failure; a population-based study. Eur Heart J 1999;20:421-28.

11. McMurray JJ, Packer M, Desai AS, et al. Angiotensinneprilysin inhibition versus enalapril in heart failure. $\mathrm{N}$ Engl J Med 2014;371:993-1004.

12. Liu RC. Focused treatment of heart failure with reduced ejection fraction using sacubitril/valsartan. Am J Cardiovasc Drugs 2018;18:473-82.

13. Sauer AJ, Cole R, Jensen BC, et al. Practical guidance on the use of sacubitril/valsartan for heart failure. Heart Fail Rev 2019;24:167-76.

14. Maggioni AP, Orso F, Calabria S, et al. The real-world evidence of heart failure: findings from 41,413 patients of the ARNO database. Eur J Heart Fail 2016; 18:402-10.

15. Marangoni E, Lissoni F, Raimondi Cominesi I, Tinelli S. Epidemiologia, impatto organizzativo e costi dello scompenso cardiaco in Italia. G Ital Cardiol 2012;13: 139-44.

16. Di Lenarda A, Scherillo M, Maggioni AP, et al. Current presentation and management of heart failure in cardiology and internal medicine hospital units: A tale of two worlds - The TEMISTOCLE study. Am Heart J 2003;146:e12:1-10.

17. Pérez-Belmonte LM, Zafra FJ, Pérez-Diaz JM, et al. Heart failure in internal medicine and cardiology: epidemiological and clinical characteristics. Eur J Intern Med 2013;24:e16.

18. Petrovic V, Ibrahim S, Palda VA. A tale of two specialties: differences between heart failure patients admitted to internal medicine and cardiology. CJGIM 2013;8:4-7.

19. Verdiani V, Panigada G, Fortini A, et al. The heart failure in internal medicine in Tuscany: the SMIT Study. Ital J Med 2015;9:349-55. 
20. Ponikowsky P, Voors AA, Anker SD, et al. 2016 ESC Guidelines for the diagnosis and treatment of acute and chronic heart failure. Eur Heart J 2016; doi:10.1093/eurheartj/ehw128.

21. Yancy CW, Jessup M, Bozkurt B, et al. 2017 ACC/AHA/HFSA Focused update of the 2013 ACCF/AHA guideline for the management of heart failure. Circulation. 2017;136:e137-61.

22. Jhund PS, McMurray JJV. The neprilysin pathway in heart failure: a review and guide on the use of sacubitril/valsartan. Heart 2016;102:1342-47.

23. Jhund PS, Fu M, Bayrman E, Chen CH, et al. Efficacy and safety of LCZ696 (sacubitril-valsartan) according to age: insights from PARADIGM-HF. Eur Heart J 2015;36:2576-84.

24. Lewis EF, Claggett BL, McMurray JJV, et al. Healthrelated quality of life outcomes in PARADIGM-HF. Circ Heart Fail 2017; doi:10.1161/CIRCHEARTFAILURE.116.003430.

25. Chandra A, Lewis EF, Claggett BL, et al. Effects of sacubitril/valsartan on physical and social activity limitations in heart failure patients: a secondary analysis of the PARADIGM-HF Trial. JAMA Cardiol 2018;3:498-505.

26. Heywood JT, Fonarow GC, Costanzo MR, et al. High prevalence of renal dysfunction and its impact on outcome in 118,465 patients hospitalized with decompensated heart failure: a report from the ADERE database. J Cardiac Fail 2007;13:422-30.

27. Ecder T. Renal and metabolic effects of valsartan. Kardiyol Derg 2014;14:S14-9.

28. Wong PC, Guo J, Zhang A. The renal and cardiovascular effects of natriuretic peptides. Adv Physiol Educ 2017;41:179-85.

29. Voors AA, Gori M, Liu LC, et al. Renal effects of the angiotensin receptor neprilysin inhibitor LCZ696 in heart failure patients and preserved ejection fraction. Eur J Heart Fail 2015;17:510-7.

30. Senni M, McMurray JJV, Wachter R, et al. Initatiating sacubitril/valsartan (LCZ696) in heart failure: results of TITRATION, a double-blind, randomized comparison of two uptitration regimens. Eur J Heart Fail 2016;18: 1193-202.

31. Damman K, Gori M, Claggett B, et al. Renal effects and associated outcomes during angiotensin-neprilysin inhibition in heart failure. $\mathrm{J}$ Am Coll Cardiol HF 2018;6:489-98.

32. Biagi P, Gussoni G, Iori I, et al. Clinical profile and predictors of in-hospital outcome in heart failure patients. The FADOI-CONFINE Study. Int J Cardiol 2011;152: 88-94.

33. Seferovic JP, Claggett B, Seidelmann SB, et al. Effect of sacubitril/valsartan versus enalapril on glycaemic control in heart failure patients and diabetes: a post-hoc analysis from the PARADIGM-HF trial. Lancet Diabetes Endocrinol 2017;5:333-40.

34. Birkenfeld AL, Boschmann M, Moro C, et al. Lipid mobilization with physiological atrial natriuretic peptide concentrations in humans. J Clin Endocrinol Metab 2005;90:3622-28.

35. Birkenfeld AL, Budziarek P, Boschmann M, et al. Atrial natriuretic peptide induces postprandial lipid oxidation in humans. Diabetes 2008;57:3199-204.

36. Coué M, Badin PM, Vila IK, et al. Defective natriuretic peptide receptor signaling in skeletal muscle links obesity to type 2 diabetes. Diabetes 2015;64:4033-45.

37. Engeli S, Birkenfeld AL, Badin PM, et al. Natriuretic peptides enhance the oxidative capacity of human skeletal muscle. J Clin Invest 2012;122:4675-9.

38. Gheorghiade M, Vaduganathan M, Ambrosy A, et al. Current management and future directions for the treatment of patients hospitalized for heart failure with low blood pressure. Heart Fail Rev 2013;18:107-22.

39. Böhm M, Young R, Jhund PS, et al. Systolic blood pressure, cardiovascular outcomes and efficacy and safety of sacubitril/valsartan (LCZ696) in patients with chronic heart failure and reduced ejection fraction: results from PARADIGM-HF. Eur Heart J 2017;38:1132-43.

40. Vardeny O, Claggett B, Kachadourian J, et al. Incidence, predictors, and outcomes associated with hypotensive episodes among heart failure patients receiving sacubitril/valsartan or enalapril: the PARADIGM-HF Trial. Circ Heart Fail 2018;11:e004745.

41. Vardeny O, Claggett B, Kachadourian J, et al. Reduced loop diuretics use in patients taking sacubitril/valsartan compared with enalapril: the PARADIGM-HF trial. Eur J Heart Fail 2019;21:337-341.

42. Cermakova P, Eriksdotter M, Lund LH, et al. Heart failure and Alzheimer's disease. J Intern Med 2015;277: 406-25.

43. Vodovar N, Paquet C, Mebazaa A, et al. Neprilysin, cardiovascular, and Alzheimer's desease: the therapeutic split? Eur Heart J 2015;36:902-5.

44. McMurray JJ, Packer M, Solomon SD. Neprilysin inhibition for heart failure. N Engl J Med 2014;371:2336-7.

45. Patel N, Gluck J. Is Entresto good for the brain? World J Cardiol 2017;9:594-9.

46. Cannon JA, McMurray JJ, Quinn TJ. "Heart and minds": association, causation and implication of cognitive impairment in heart failure. Alzheimer Res Ther 2015;7:22.

47. Pandharipande PP, Girard TD, Jackson JC, et al. Longterm cognitive impairment after critical illness N Engl J Med 2013;369:1306-16.

48. Albert NM, Yancy CW, Liang L, et al. Use of aldosterone antagonists in heart failure. JAMA 2009;302: 1658-65.

49. Hernandez AF, Mi X, Hammill BG, et al. Associations between aldosterone antagonist therapy and risks of mortality and readmission among heart failure patients and reduced ejection fraction. JAMA 2012;308:2097-107.

50. Bress AP, King JB, Brixner D, et al. Pharmacotherapy treatment patterns, outcomes, and health resource utilization among heart failure patients with reduced ejection fraction at a U.S. Academic Medical Center. Pharmacotherapy 2016;36:174-86.

51. Ferreira JP, Rossignol P, Machu JL, et al. Mineralcorticoid receptor antagonist pattern of use in heart failure with reduced ejection fraction: findings from BIOSTATCHF. Eur J Heart Fail 2017;19:1284-93.

52. Patterson SJ, Reaves AB, Tolley EA, et al. Underutilization of aldosterone antagonists in heart failure. Hosp Pharm 2017;52:698-703.

53. Fortini A, Verdiani V, Panigada G, et al. Triple therapy at discharge from internal medicine wards in heart failure patients with reduced ejection fraction: results from an observational study. Clin Ter 2018;169:e287-91. 
54. Juurlink DN, Mamdani MM, Lee DS, et al. Rates of hyperkalemia after publication on the randomized aldactone evaluation study. N Engl J Med 2004;351:543-51.

55. Palmer BF. Managing hyperkalemia caused by inhibitors of the renin-angiotensin-aldosterone system. N Engl J Med 2004;351:585-92.

56. Desai AS, Vardeny O, Claggett B, et al. Reduced risk of hyperkalemia during treatment of heart failure with mineralcorticoid receptor antagonists by use of sacubitril/valsartan compared with enalapril. A secondary analysis of the PARADIGM-HF Trial. JAMA Cardiol 2017;2:79-85.

57. Jankowska EA, Ponikowska B, Majda J, et al. Hyperuricaemia predicts poor outcome in patients with mild to moderate chronic heart failure. Int $\mathrm{J}$ Cardiol 2007;115:151-5.

58. Palazzuoli A, Ruocco G, Pellegrini M, et al. Prognostic significance of hyperuricaemia in patients with acute heart failure. Am J Cardiol 2016;117:1616-21.

59. Borghi C, Cosentino ER, Rinaldi ER, Cicero AF. Uricaemia and ejection fraction in elderly heart failure outpatients. Eur J Clin Invest 2014;44:573-8.
60. Mogensen UM, Køber L, Jhund PS, et al. Sacubitril/valsartan reduces serum uric acid concentration, an independent predictor of adverse outcomes in PARADIGM-HF. Eur J Heart Fail 2018;20:514-22.

61. Vardeny O, Claggett B, Packer M, et al. Efficacy of sacubitril/valsartan vs enalapril at lower than target doses in heart failure with reduced ejection fraction: the PARADIGM-HF trial. Eur J Heart Fail 2016;18:1228-34.

62. Okumura N, Jhund PS, Gong J, et al. Effects of sacubitril/valsartan in the PARADIGM-HF Trial (prospective comparison of ARNI with ACEI to determine impact on global mortality and morbidity in heart failure) according to background therapy. Circulation Heart Fail 9:e003212.

63. Velazquez EJ, Morrow DA, DeVore AD, et al. Angiotensin- Neprylisin inhibition in acute decompensated heart failure. N Engl J Med 2019;380:539-48.

64. Wachter R, Senni M, Belohlavek J, et al. Initiation of sacubitril/valsartan in hospitalized heart failure patients with reduced ejection fraction after hemodynamic stabilization: primary results of the TRANSITION study. Eur Heart J 39;Suppl1:886. 